maintained and interpreted with the utmost rigour, auxin might instead permit development, within an apical or basal context set by additional input. At least we are now in a position to muse on these possibilities, thanks to the work of Friml and colleagues.

Stefan Kepinski and Ottoline Leyser are in the Department of Biology, University of York, York YO10 5YW, UK. e-mails: ssk2@york.ac.uk hmol1@york.ac.uk
1. Wolpert, L. Principles of Development (Oxford Univ. Press, 2002).

2. Jürgens, G. EMBO J. 20, 3609-3616 (2001).

3. Friml, J. et al. Nature 426, 147-153 (2003).

4. Berleth, T. \& Sachs, T. Curr. Opin. Plant Biol. 4, 57-62 (2001).

5. Kepinski, S. \& Leyser, O. Plant Cell 14, S81-S95 (2002).

6. Friml, J. Curr. Opin. Plant Biol. 6, 7-12 (2003).

7. Hadfi, K., Speth, V. \& Neuhaus, G. Development 125, 879-887 (1998).

8. Hardtke, C. S. \& Berleth, T. EMBO J. 17, 1405-1411 (1998).

9. Hamann, T. et al. Genes Dev. 16, 1610-1615 (2002)

10. Steinmann, T. et al. Science 286, 316-318 (1999).

11. Geldner, N. et al. Cell 112, 219-230 (2003).

Condensed-matter physics

\title{
The quest for imperfection
}

Thomas F. Rosenbaum

The electrical properties of silver chalcogenides are unusually affected by magnetic fields. A simulation suggests how this might arise from tiny imperfections and could facilitate the design of new materials.

$\mathrm{T}$ he change in the electrical resistance of a material with magnetic field — its magnetoresistance - lies at the heart of many applications, from storing information in a computer to measuring the speed of a car. The resistance of two materials in particular, silver selenide and silver telluride, can be made to increase linearly as the applied magnetic field increases, up to field values at least one million times that of the Earth's magnetic field ${ }^{1,2}$. This linear response over such a large range makes these semiconductor materials attractive as magnetic-field sensors, although their performance is unusual in terms of basic physics. Parish and Littlewood $^{3}$ have tackled the question, as they report on page 162 of this issue, using a combination of fundamental theory and computer simulations. They show that it is not material perfection, but macroscopic disorder and inhomogeneity, that are the essential ingredients for such a remarkable magnetoresistive response.

Crystalline perfection and purity is the paradigm for the semiconductor electronics industry, where gowned technicians in dustfree clean rooms prepare crystal wafers of silicon containing impurities controlled to one part in a billion. In contrast, perfectly stoichiometric, homogeneous silver selenide and silver telluride $\left(\mathrm{Ag}_{2} \mathrm{Se}\right.$ and $\mathrm{Ag}_{2} \mathrm{Te}$, respectively, and 'silver chalcogenides' collectively) are useless for applications. It is only when a small amount of excess silver is introduced, ideally at the level of one part in 10,000, that the material becomes interesting. The exact distribution of the excess silver atoms in the 'optimally doped' material is not known, but the atoms presumably group in small clusters or fine wires on nanometre or micrometre scales.

So how do such fluctuations in composition lead to an anomalously large, linear magnetoresistance that also doesn't saturate at high field values? Abrikosov ${ }^{4}$ was the first to realize that the problem should be modelled in terms of the creation of an 'effective medium', in which disorder is combined with quantum mechanics in a theory that explains the properties of semiconductors that are almost, but not quite, metals. Parish and Littlewood ${ }^{3}$, however, invoke a classical explanation that is applicable over a broad range of temperatures and to a wide variety of compositional irregularities. They construct (conceptually, and on the computer) a two-dimensional, random network of resistors - effectively, an inhomogeneous semiconductor. Usually, a resistor has a single current input and output, but these resistors are special: each disk-like resistor has four terminals around its edge, two for the input and output of current and two additional posts for reading voltages. In this way, the voltage drop across the resistor need not be aligned with the direction of the current. From such a four-terminal device it is possible to construct a matrix of the device's conductivity: diagonal components correspond to the voltage being aligned with the current; off-diagonal components arise because a current in one direction can induce a voltage in a different direction.

There are two key elements to considerthe effect of an applied magnetic field, and the response of the resistor network as a whole. When a magnetic field is applied perpendicular to the direction of current flow, the charge-carrying electrons in each disk are forced into circular trajectories (called cyclotron orbits) and a voltage is induced that is transverse to both the field and the current directions. This transverse voltage is known as the Hall voltage and has the special property of growing linearly with increasing magnetic field. This linear response is obviously necessary for matching theory to experiment, but it is not enough to explain the exact behaviour of the silver chalcogenides. The magnetoresistance would still be expected to saturate at a magnetic-field strength that is almost 100 times lower than that already reached in experiments experiments that show no evidence of saturation.

It is at this point that the collective behaviour of a random array of resistance disks becomes important. No longer do the cyclotron orbits alone set the definitive scale of the response. Rather, disorder and inhomogeneity, as expressed by fluctuations in the properties of the individual array resistors - for example, whether the Hall voltage is positive or negative - are the appropriate control parameters. The current paths no longer simply follow electricfield lines, but can loop back on themselves and even travel perpendicular to the applied voltage. The net effect in a large array is a linear magnetoresistance that corresponds to that of the silver chalcogenides. Moreover, Parish and Littlewood's approach in essence presents design rules for creating new materials with a tailor-made magnetoresistive response.

The concept of exploiting imperfection can be applied across the field of condensed-matter physics. Heterogeneous systems, from polymers to high-temperature superconductors, organize themselves on microscopic length scales with macroscopic consequences. Eschewing the rigorous demands and expense of perfectly replicated and ordered devices has obvious advantages, but introduces complications in addressability and control. A good example is the nascent field of quantum computation. Here it is feared that disorder will lead to dissipation, disrupting the finely tuned superposition of quantum states that is necessary for processing quantum information. Yet quantum entanglement can, in fact, dominate the magnetic response of a solid-state system ${ }^{5}$.

Rather than building gates between components in a conventional sense, it may be possible to take advantage of materials that have different microscopic environments, and which are tuned and addressable electronically, magnetically or optically. Following this strategy, disorder will be sought for its natural mix of physical parameters and length scales, permitting the development of flexible and easily scalable devices.

Thomas F. Rosenbaum is in the Department of

Physics and the James Franck Institute,

University of Chicago, 5640 Ellis Avenue,

Chicago, Illinois 60637, USA.

e-mail:t-rosenbaum@uchicago.edu

1. Xu, R. et al. Nature 390, 57-60 (1997).

2. Husmann, A. et al. Nature 417, 421-424 (2002).

3. Parish, M. M. \& Littlewood, P. B. Nature 426, 162-165 (2003).

4. Abrikosov, A. Phys. Rev. B 58, 2788-2794 (1998).

5. Ghosh, S., Rosenbaum, T. F., Aeppli, G. \& Coppersmith, S. N. Nature 425, 48-51 (2003). 